\title{
Monozygotic Twin Pregnancy Associated with In Vitro Fertilization: Report and Revision of Three Cases Presented in a Clinic at 10,925 Feet Above Sea Level
}

\author{
Luis Vargas Tominaga ${ }^{1}$, Ricardo Pella Cáceres ${ }^{1}$, Alberto Vargas Lechuga ${ }^{1}$, Livia Bartolo Durán ${ }^{1}$, Mariela Serrano \\ Vargas $^{1}$ \\ ${ }^{1}$ Centro de Fertilidad y Ginecología del Sur, Cusco, Peru \\ Presented as a poster in the Cusco Regional Hospital Golden Anniversary Congress - Cusco, Peru - May, 2014.
}

\begin{abstract}
3 cases of monozygotic twin pregnancies associated with IVF presented at Centro de Fertilidad y Ginecología del Sur. In this study, we describe the cases and review the literature, noting possible related factors.
\end{abstract}

Keywords: Monozygotic, IVF, ICSI, IVF, MZT.

\section{INTRODUCTION}

Monozygotic twin pregnancy (MZT) can be found in 0.40 to $0.45 \%$ in the general population and $30 \%$ in twin pregnancies (Aston et al., 2008). It's a difficult situation, because 10 to $20 \%$ of patients with MZT are at a high risk of having twin-to-twin transfusion syndrome, with asymmetric fetal growth and with a fetal mortality of $80 \%$ or more in untreated cases (Harkness \& Crombleholme, 2005). In monoamniotic MZT cases there is a great risk of umbilical cord entanglement.

With the development of assisted reproduction, twin and multiple births are associated conditions, representing 20 to $30 \%$ of the pregnancies obtained through this method. In 2011, the Latin American Assisted Reproduction Network reported $21 \%$ of twin pregnancies and $1.3 \%$ of triplets or more fetuses per cycle through in vitro fertilization (IVF) (Zegers-Hochschild et al., 2013).

In 1984 the first MZT associated to IVF was reported (Yovich et al., 1984); and from that report, MZT associated with IVF has been observed in a frequency between 1 to 3\% (Aston et al., 2008; Saito et al., 2000; Papanikolaou et al., 2010; Kawachiya et al., 2011).

This report describes three cases of IVF-related MZT found in the Centro de Fertilidad y Ginecología del Sur (CFGS), in Cusco, located at 10,925 feet above sea level (f.a.s.I.); we discuss their evolution and possible factors related to the development of MZT.

\section{CLINICAL CASES \\ CASE 1}

A woman aged 37, with 12 years of primary infertility underwent three surgeries, two for endometriosis and one for fibroids. Her husband is 40 years old with diabetes under treatment and with a normal semen analysis. Two years before, they underwent an IVF treatment in another center, transferring two embryos unsuccessfully. On the same year, they underwent a first IVF cycle at CFGS, two embryos were transferred and a biochemical pregnancy was achieved. One year later, they were submitted to a second IVF cycle, transferring two embryos with a negative result.

In the third cycle, the patient was given metformin and the ovaries were stimulated with 225 IU of HMG from the first to the fifth day of the cycle, associated with $0.5 \mathrm{mg}$ of leuprolide acetate, from day 21 of the previous cycle to one day before follicle aspiration. Then, recombinant human chorionic gonadotropin (hCG) was administered in the ninth day and 34 hours after the follicle was aspirated. We obtained one mature oocyte and performed Intracytoplasmic Sperm Injection (ICSI).

On the third day of culture an embryo in the 8-cell stage and grade II was transferred and we recommended luteal support with $800 \mathrm{mg}$ of micronized progesterone. Sixteen days after follicle aspiration, we obtained $189 \mathrm{mIU} / \mathrm{ml}$ of $\beta$-hCG. An ultrasound scan showed a twin monochorionic diamniotic pregnancy with both embryos active.

The pregnant woman had hyperemesis gravidarum in the first three months and intrauterine growth retardation in one of the fetus since the $19^{\text {th }}$ week.

Upon 36 weeks, two healthy girls were born through caesarean section, one of them weighing 2,500 grams and the other with 1,500 grams.

\section{CASE 2}

A 46 year-old woman with 6 years of primary infertility with bilateral tubal obstruction due to hydrosalpinx. Her husband, 44 years old, healthy, had normal semen analysis. They were submitted to an IVF cycle in another center, at first with fresh, and then with frozen embryos, and achieved a biochemical pregnancy.

At CFGS, she received a single dose of $3,75 \mathrm{mg}$ of leuprolide acetate on the $21^{\text {st }}$ day of the previous $\mathrm{cy}$ cle. Then, her period started, she used 2 to $8 \mathrm{mg}$ of oral estradiol valerate to thicken the endometrial line to $7 \mathrm{~mm}$ on the $11^{\text {th }}$ day of the cycle. On the $16^{\text {th }}$ day of the cycle, we started luteal support with a vaginal ring of progesterone and $600 \mathrm{mg}$ of vaginal micronized progesterone.

The follicles were then aspirated on the next day, from an anonymous donor aged 26, and we did the ICSI with the patient's husband sperm. On the fifth day of culture, 2 blastocysts grade AA were transferred, according to the classification of Gardner et al. (2007) (Figure 1).

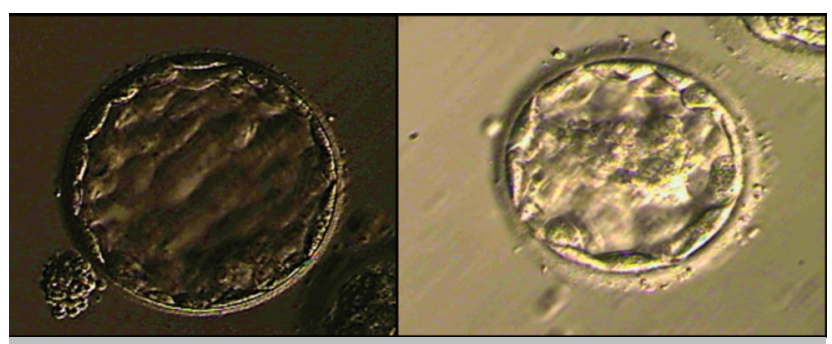

Figure 1: Expanded blastocysts grade AA 
Eighteen days after ICSI we assessed B-hCG level, which resulted in $339 \mathrm{mIU} / \mathrm{ml}$. The ultrasound scan showed a triplet dichorionic diamniotic pregnancy, with one of the sacs constituted by a twin monochorionic monoamniotic pregnancy.

Upon week 11.5 we did a cervix cerclage. The pregnancy developed with symmetric growing of the three fetuses until the $29^{\text {th }}$ week, when intra-uterine growth retardation was observed.

Upon 34 weeks, three healthy children were born through caesarean section. The first baby girl weighed 1,850 grams and the other two male babies weighed 1,500 grams and 1,370 grams each, respectively.

\section{CASE 3}

A 37 year-old woman with 5 years of secondary infertility. She previously had one normal delivery and two surgeries for endometriosis and a third for uterine fibroids.

Her evaluation showed right tubal obstruction. Her husband, aged 35, healthy, had normal semen analysis. Two years before they were submitted to IVF in another center, where two embryos were unsuccessfully transferred.

She was given a single dose of $3.75 \mathrm{mg}$ of leuprolide acetate on the $21^{\text {st }}$ day of the previous cycle. Then, her period started, she used 2 to $6 \mathrm{mg}$ of estradiol valerate, to thicken the endometrial lining to $7.5 \mathrm{~mm}$ on the $11^{\text {th }}$ day of the cycle. On the $15^{\text {th }}$ day of the cycle we started luteal support with a vaginal ring of progesterone and $600 \mathrm{mg}$ of vaginal micronized progesterone. On the next day we aspirated the follicles from an anonymous donor aged 26 and we did an ICSI sperm from the patient's husband sperm. On the third day of culture, 3 embryos were transferred: 10 -cell stage grade I; 8 -cell stage grade II; 6-cell stage grade I. We vitrified 3 embryos: 8 -cell stage grade III; 7 -cell stage grade III; 7-cell stage grade II. After 17 days of ICSI the $\beta$-hCG result was negative.

Five months later we devitrified and transferred 3 embryos, following the same procedure as aforementioned. The dose of estradiol valerate started at $4 \mathrm{mg}$ and went up to $8 \mathrm{mg}$, making it possible for the endometrium to reach $9 \mathrm{~mm}$ on the $11^{\text {th }}$ day of the cycle. We started luteal support on the $14^{\text {th }}$ day of the cycle and then we devitrified and transferred the embryos on the $18^{\text {th }}$ day of the cycle (Figures 2, 3 and 4). Twenty days after luteal support onset the $\beta$-hCG was at $391 \mathrm{mIU} / \mathrm{ml}$. Ultrasound scan showed a monochorionic monoamniotic twin pregnancy.

The pregnancy was favorable until the $11^{\text {th }}$ week of gestation. Heart activity was negative in the first fetus at the $12^{\text {th }}$ week, and in the second at the $14^{\text {th }}$ week, requiring induction and manual intrauterine suction.

\section{DISCUSSION}

At the CFGS, the 3 cases of MZT came among 573 transfers $(0.52 \%)$ and from 192 clinical pregnancies $(1.59 \%)$; an internationally reported rate (Aston et al., 2008; Saito et al., 2000; Papanikolaou et al., 2010; Kawachiya et al., 2011).
MZT can have the following 3 presentations: dichorionic and diamniotic, when the division occur before embryonic cell differentiation, within the 4 first days of fertilization; monochorionic diamniotic when the blastocyst internal cellular mass (ICM) is divided before the implantation (cases 1 and 2); and monochorionic monoamniotic when ICM is divided after the implantation (case 3 ).

Laboratory data, including morphology of the transferred embryos in these 3 cases, was recorded through computerized images obtained on the day of the transfer. These images were analyzed and the ICM was not divided in any of the embryos transferred, and nothing out of the ordinary was found.

Upon analyzing the association between MZT and IVF, possible factors are noteworthy: age, zona pellucida manipulation, transfer at the blastocyst stage, transfer of fresh or frozen embryos and ovulation induction.

Regarding age as an MZT-associated factor, publications before 2005 mentioned that IVF in women older than 35 years of age have a tendency for this event. They proposed a subtle thinning of the zona pellucida with aging, which could allow asymmetric lysis with multiple output sites during blastocyst hatching (Cohen et al., 1992). However, a recent study by Knopman et al. (2010), analyzed variables that suggested a risk to MZT, it was only at the age under 35 that there was a significant difference $(2.01 \%)$ in the risk of developing IVF-related MZT. Similarly, Kawachiya et al. (2011) stressed a greater frequency of MZT in younger patients. The ages of oocyte origin in the 3 cases presented in the CFGS were 37, 26 and 26, respectively.

Zona pellucida manipulation is another factor considered associated with MZT. It is done in ICSI, assisted hatching $(\mathrm{AH})$ and blastomere biopsy for preimplantation genetic diagnosis (PGD). It is argued that artificial rupture of the zona pellucida in these procedures results in the presence of an orifice with subsequent herniation of the blastomeres, and blastocyst expansion may result in a mechanical unfolding of the embryo, resulting in MZT (Talansky \& Gordon, 1988; Van Langendonckt et al., 2000). Saito et al. (2000) reported 9 MZT from a total of 279 pregnancies $(3.2 \%)$, with an increased risk in cases where manipulation originated wider rupture of the zona pellucida. Tarlatzis et al. (2002) reported 6 MZT from 102 ICSI cycles $(5.9 \%)$ and nothing from 79 conventional IVF cycles. Alikani et al. (2003) found MZT in $2.4 \%$ of ICSI cycles in contrast to $1.6 \%$ in conventional IVF cycles. Abusheika et al. (2000) reported that there is a higher incidence of MZT in the group of patients who were treated with ICSI $(8.9 \%)$ when compared with conventional IVF (0.9\%). Knopman et al. (2010) found no significant difference in the incidence of MZT $(2.0 \%)$ in their study group when ICSI was performed in 1410 cycles or IVF in 3039 cycles. Kawachiya et al. (2011), in a retrospective study of 47,841 patients with transfer of a selected single embryo (SET), also did not find differences between the groups. At CFGS, the 3 cases of MZT occurred in patients in whom ICSI was per-
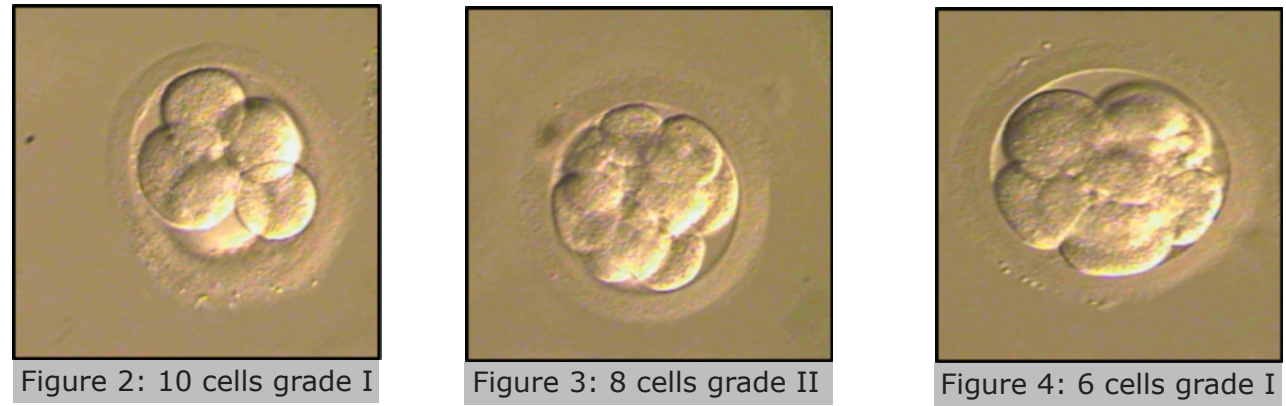
formed, from a total of 484 embryo transfers $(0.62 \%)$ and 173 pregnancies achieved $(1.73 \%)$ using this technique. There has not been MZT associated with conventional IVF (89 transfers and 19 pregnancies).

It has been mentioned that prolonged embryo culture until the blastocyst stage is another factor associated with MZT. It is suggested that the low concentration of calcium in the prolonged culture medium breaks intercellular junctions in the ICM and predisposes for embryo division (Steinman \& Valderrama, 2001). Kawachiya et al. (2011) observed a rate of $1.01 \%$ of MZT in 14,956 pregnancies achieved through SET, finding statistical differences between $0.61 \%$ incidence of MZT when the transfer is done at cleavage stage and $1.21 \%$ when in the blastocyst stage. Papanikolaou et al. (2010) found $2.2 \%$ of MZT in 579 cases, $2.6 \%$ when the transfer is at cleavage stage and $1.8 \%$ when at blastocyst stage, although the differences were not statistically significant.

Chang et al. (2009), in a meta-analysis found association between MZT and blastocyst transfer. However, he noted that in studies published after 2002, there was no significant difference between the transfer of cleavage stage embryos or blastocysts and the incidence of MZT. They argue that the improvement in the conditions of prolonged cultures would lead to the lower frequency of MZT. Another meta-analysis by Moayeri et al. (2007) also found statistically significant differences, with an incidence of $0.41 \%$ MZT when the transfer is at cleavage stage and $1.64 \%$ when it is at blastocyst stage. They observed a decrease in the incidence of MZT associated with blastocyst transfer over a period of eight years, suggesting that this might be related to improvements in embryo transfer techniques. This year, in the United States, the latest report by the Society for Assisted Reproductive Technology (SART) stated that MZT risk increases with blastocyst transfer; however, there is a greater effect of $\mathrm{AH}$ in the development of this event, particularly in embryos transferred at cleavage stage (Luke et al., 2014).

Ovulation induction is also mentioned as a possible MZT-related factor. A twin pregnancies prospective study performed in East Flanders found an MZT rate of $1.2 \%$ after ovarian stimulation, about a 3 times higher incidence than in spontaneously conceived pregnancies (Derom et al., 1987). A recent study by the same group revealed a high proportion of MZT after ovarian stimulation with clomiphene citrate (Derom et al., 2006). They argued that the use of ovulation induction could cause inconsistent hardening of the zona pellucida, which could cause some weak points through which the blastocyst could herniate. However, Kawachiya et al. (2011) did not find differences in MZT events among patients who used clomiphene citrate and those who had spontaneous cycles. Schachter et al. (2001) found greater association with the use of gonadotropins $(1.5 \%)$ than with other assisted reproduction methods (0.95\%). SART, as a result of univariate analysis, mentioned in its communication this year, that ovulation disorders, oocyte donation and use of agonists may increase the risk of MZT (Luke et al., 2014).

\section{CONCLUSIONS}

The evaluation of the 3 cases presented by the CFGS reaffirms MZT as a high-risk condition. The literature review, in contrast with our observation at the CFGS, allows us to take into consideration the age and the zona pellucida manipulation as the factors most related to MZT in association with IVF.

\section{CONFLICT OF INTERESTS}

No conflict of interest have been declared.

\section{Corresponding author:}

Luis Vargas Tominaga

Centro de Fertilidad y Ginecología del Sur (CFGS)

Cuzco, Peru

E-mail: luisvargastominaga@hotmail.com

\section{REFERENCES}

Abusheika N, Salha O, Sharma V, Brinsden P. Monozygotic twinning and IVF/ICSI treatment: a report of 11 cases and review of literature. Hum Reprod Update 2000;6:396-3.

Alikani M, Cekleniak N, Walters E, Cohen J. Monozygotic twinning following assisted conception: an analysis of 81 consecutive cases. Hum Reprod. 2003;18:1937-43.

Aston KI, Peterson CM, Carrell DT. Monozygotic twinning associated with assisted reproductive technologies: a review. Reproduction. 2008;136:377-86.

Chang $\mathrm{H}$, Lee J, Such C, Kim S. Impact of blastocyst transfer on offspring sex ratio and the monozygotic twinning rate: a systematic review and meta-analysis. Fertil Steril. 2009; $91: 238-90$.

Cohen J, Alikani M, Trowbridge J, Rosenwaks Z. Implantation enhancement by selective assisted hatching using zona drilling of embryos with poor prognostic. Human Reprod. 1992; 7:685-91.

Derom C, Vlietink R, Van den berghe $H$, Thiery M. Increased monozygotic twinning rate after ovulation induction. Lancet $1987 ; 1: 1236-8$.

Derom C, Leroy F, Vlientinck R, Fryns J, Derom R. High frequency of iatrogenic monozygotic twins with administration of clomiphene citrate and a change in chorionicity. Fertil Steril 2006;85:755-7.

Gardner DK, Stevens J, Sheehan CB, Schoolcraft WB. Analysis of blastocyst morphology. In: Elder K, Cohen J, eds. Human preimplantation embryo selection, Informa Uk. Ltd. 2007. p.79-88

Harkness UF, Crombleholme TM. Twin-twin transfusion syndrome: where do we go from here? Semin Perinatol. 2005;29:296-304.

Kawachiya S, Bodri D, Shimada N, Kato K, Takehara, Y, Kato O. Blastocyst culture is associated with an elevated incidence of monozygotic twinning after single embryo transfer. Fertil Steril. 2011;95:2140-42.

Knopman J, Krey L, Lee J, Fino M, Novetsky A, Noyes N. Mozozygotic twinninning, an eight-year experience at a large IVF center. Fertil Steril. 2010; 94:502-10.

Luke B, Brown M, Whatman E, Stern J. Factors associated with monozygosity in assisted reproductive technology pregnancies and the risk of recurrence using linked cycles. Fertil Steril. 2014;3:683-9

Moayeri S, Behr B, Lathi RB, Westphal LM, Milki AA. Risk of monozygotic twinning with blastocyst transfer decrease over time: an 8-year experience. Fertil Steril. 2007;87:1028-32.

Papanikolaou EG, Fatemi $\mathrm{H}$, Venetis C, Donoso P, Kolibianakis E, Tournaye H, Tarlatzis B, Devroey P. Monozygotic twinning is not increased after single blastocyst transfer compared with single cleavage-stage 
embryo transfer. Fertil Steril. 2010;93: 592-7.

Saito H, Tsutsumi O, Noda Y, Ibuki Y, Hiroi M. Do assisted reproductive technologies have effects on the demography of mozigotic twinning? Fertil Steril. 2000; 74: 178-9.

Schachter M, Raziel A, Friedler S, Strassburger D, Bern O, Ron-EI R. Monozygotic twinning after assisted reproductive techniques. A phenomenon independent of micromanipulation. Human Reprod 2001;16:1264-9.

Steinman G, Valderrama E, Mechanisms of twinning. II. Placentation calcium reduction and modified compaction. J Reprod Med 2001; 46:995-1002.

Talansky B, Gordon J. Cleavage characteristics of mouse embryos inseminated and cultured after zona pellucida drilling. Gamete Research 1988;21:277-87.
Tarlatzis B, Qublan H, Sanopoulou T, Zepiridis L, Grimbizis $\mathrm{G}$, Bontis J. Increase in the monozygotic twinning rate after intracytoplasmic sperm injection and blastocyst stage embryo transfer. Fertil Steril. 2002;77:196-8.

Van Langendonckt A, Wyns C, Godin P, Toussaint-Demylle D, Donnez J. Atypical hatching of a human blastocyst leading to monozygotic twinning: a case report. Fertil Steril. $2000 ; 74: 1047-50$.

Yovich JL, Stanger JD, Grauaug A, Barter RA, Lunay G, Dawkins RL, Mulcahy MT. Monozygotic twins from in vitro fertilization. Fertil Steril. 1984;41:833-7.

Zegers-Hochschild F, Schwarze JE, Crosby JA, Musri C, de Souza MCB. Assisted reproductive technologies (ART) in Latin America: The Latin American Registry, 2011. JBRA Assist Reprod. 2013; 17:216-23 